\title{
Promotion of physical activity and healthy food choices was hampered by competitiveness, lack of quality food, easy access to non-nutritious food, and time constraints
}

Bauer KW, Yang YW, Austin SB. "How can we stay healthy when you're throwing all of this in front of us?" Findings from focus groups and interviews in middle schools on environmental influences on nutrition and physical activity. Health Educ Behav $2004 ; 31: 34-46$.

\section{What are the factors in school social and physical environments that support or interfere with the promotion of healthy nutrition and physical activity?}

\section{DESIGN}

Qualitative study.

\section{SETTING}

Grade 7 and 8 classes (children 12-14 y) in 2 public schools (500-800 students) in northeastern USA.

\section{PARTICIPANTS}

26 students and 23 faculty and staff members.

\section{METHODS}

Students were divided by grade and sex into 7 focus groups, and faculty and staff members were divided into 3 focus groups. 10 individual interviews were conducted with key informants including administrators, cafeteria managers, physical education directors, school nurses, guidance counsellors, and health educators. Focus groups and individual interviews lasted 45-60 minutes. Topics included the physical and social environments in the schools and how the environments influenced students' nutrition and physical activity choices. Focus groups and interviews were audiotaped, transcribed verbatim, and analysed using the grounded theory approach. Concepts, categories, and themes were identified using an iterative process.

\section{MAIN FINDINGS}

Both schools offered physical education classes, school sports teams, and open gym time before the start of classes. However, students and staff described competitiveness and time and safety as significant barriers to physical activity. Competitiveness: athletic competitiveness occurred in physical education classes and open gym periods, prompting children who were overweight or less athletically skilled not to participate. Girls were less likely to make use of open gym time than boys, who tended to dominate the gym. Lack of coaches, playing space, and equipment meant that extracurricular sports teams involved only the most athletically skilled children. Time and safety: walking and riding bicycles to and from school were not promoted because of safety issues (ie, heavy traffic and encounters with strangers) and lack of time.

Barriers to healthy nutrition focused on the quality of food served in the school cafeterias, accessibility of snack foods in vending machines, time limitations, and teasing and weight concerns. Quality of food: although cafeteria food was required to meet minimum national nutrition standards, many described it as greasy and high in fat, with few vegetable and fruit choices available. Vending machines:

For correspondence: Dr S B Austin, Division of Adolescent and Young Adult Medicine, Children's Hospital Boston, Boston, MA, USA. bryn.austin@ tch.harvard.edu

Sources of funding: Boston Obesity Nutrition Research Center; Massachusetts Health Research Institute; McCarthy Family Foundation; Maternal and Child Health Bureau, US Department of Health and Human Services. vending machines with non-nutritious snacks and sugar sweetened drinks were readily available. However, school administrators indicated that the money made from the vending machines subsidised school trips and scholarships. Time limitations: students and staff indicated that lunch periods were so short that buying food from a vending machine was often a preferred option to waiting in line for cafeteria food. Teasing and weight concerns: because of the large number of students in the cafeteria at one time, teasing of overweight children or criticism of food choices often went unnoticed by teacher monitors. Girls tended to talk about dieting and losing weight. Girls also described feeling uncomfortable eating in public, particularly in front of boys, because they felt unattractive.

\section{CONCLUSION}

Barriers in the school environment including competitiveness and lack of nutritious food and time to eat it hampered physical activity and healthy nutrition in school children aged $12-14$ years.

\section{Commentary}

Diabir rever evention of childhood obesity and associated diseases (eg, type 2 diabetes and cardiovascular disease) is important worldwide. Limited evidence exists, however, on which to base a programme of interventions. ' Schools have a potentially important role in promoting a healthy diet and exercise. ${ }^{2}$ The study by Bauer et al raises issues that should be considered in developing interventions in schools.

Baver et al highlight the importance of understanding culture through their use of an ecological model to examine factors that facilitate or compete with programmes and policies developed to improve physical activity and nutrition in schools. The authors explain why children and adolescents might not make use of available resources to maintain a healthy lifestyle, including physical education, sports teams, morning open gym, and healthy food in the cafeteria. It is also noteworthy that Baver et al involved children and school staff members to obtain a comprehensive view of what occurs within the school environment. Schools could be an important setting for interventions to prevent childhood obesity. However, families also play an important role, and as the authors suggest, a limitation of this study is that parents' perspectives were not solicited.

The study identifies barriers such as athletic competitiveness, teasing and bullying, time constraints, safety, weight concerns, unpalatable healthy food, and availability of non-nutritious snacks that should be addressed when developing interventions.

Olga López de Dicastillo, RN, MSc University of Navarra Pamplona, Navarra, Spain 1 Campbell K, Waters E, O'Meara S, et al. Interventions for preventing obesity in children. Cochrane Database Syst Rev 2002;(2):CD001871.

2 Casey L, Crumley E. Addressing childhood obesity: the evidence for action. Ottawa: Canadian Association of Paediatric Health Centres, Pediatric Chairs of Canada, Institute of Nutrition Metabolism and Diabetes of the
Canadian Institutes of Health Research, 2004. http://www. caphc.org/ childhood_obesity/obesity_report.pdf 